\title{
Narrow-band imaging with magnification and the water immersion technique: a case-finding, cost-effective approach to diagnose villous atrophy
}

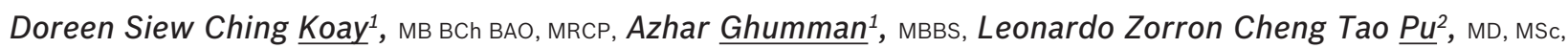
Rajvinder $\underline{\text { Singh }}^{1,2}$, FRCP, FRACP

\begin{abstract}
INTRODUCTION Narrow-band imaging with magnification endoscopy (NBI-ME) allows real-time visual assessment of the mucosal surface and vasculature of the gastrointestinal tract. This study aimed to determine the performance of NBI-ME combined with the water immersion technique (NBI-ME-WIT) in detecting villous atrophy.

METHODS All patients who underwent gastroscopy were included. The duodenum was further examined with NBI-MEWIT only after examination with white light endoscopy did not reveal a cause of anaemia or dyspepsia. Targeted biopsies were taken of visualised areas. NBI-ME-WIT findings were compared with the final histopathological analysis. We calculated the sensitivity (Sn), specificity (Sp), positive predictive value (PPV) and negative predictive value (NPV) of NBI-ME-WIT in detecting villous atrophy and the hypothetical cost saved by using a biopsy-avoiding approach.

RESULTS 124 patients (83 female) with a mean age of 46 (range 18-82) years were included. The most common indication for gastroscopy was abdominal pain (39\%), followed by anaemia (35\%), chronic diarrhoea/altered bowel habits (19\%) and dyspepsia (6\%). NBI-ME-WIT was able to detect all nine patients with villous atrophy - eight patchy and one total villous atrophy. The Sn, Sp, PPV and NPV of NBI-ME-WIT in detecting villous atrophy were $100.0 \%, 99.1 \%, 90.0 \%$ and $100.0 \%$, respectively. Taking into account the cost of biopsy forceps (AUD 17) and pathology (AUD 140), this biopsy-avoidance strategy could have saved AUD 18,055 in these patients.

CONCLUSIONS NBI-ME-WIT is a specific and sensitive tool to recognise and accurately diagnose villous atrophy. Biopsies can be avoided in patients with normal-sized villi, which may decrease the overall cost of the procedure.
\end{abstract}

Keywords: coeliac disease, narrow-band imaging, villous atrophy, water immersion technique

\section{INTRODUCTION}

Coeliac disease $(C D)$ is an immune-mediated condition of the small intestine that is characterised by mucosal inflammation, crypt hyperplasia and villous atrophy arising from exposure to dietary gluten in predisposed individuals, which resolves with avoidance of gluten in the diet. The prevalence of CD has been increasing in recent decades. ${ }^{(1,2)} \mathrm{A}$ study from Australia estimated that the disease affects $1.2 \%-1.9 \%$ of the population. ${ }^{(3)}$ Recognising CD is challenging and at times results in a delay of diagnosis..$^{(4,5)}$ $\mathrm{CD}$ is usually suspected when symptomatology, such as irondeficiency anaemia and chronic diarrhoea, is associated with malabsorption of nutrients. Following clinical suspicion, serology is generally performed to look for specific antibodies, including tissue transglutaminase immunoglobin A (IgA), anti-endomysial IgA and anti-deamidated gliadin peptide IgA antibodies. Although the antibody tests have high sensitivity (Sn) and specificity (Sp) for $\mathrm{CD}$, a definitive diagnosis relies on histological examination of biopsies from the duodenum. ${ }^{(6)}$

Normal duodenal mucosa has finger-like villi and a regular network of capillaries. Reduced duodenal folds, scalloping of fold margins, a mosaic pattern of mucosa and grooves in the mucosa are the conventional signs seen on white light endoscopy (WLE) in patients with CD. ${ }^{(7-11)}$ Histologically, villous atrophy is graded using the Marsh classification, which utilises the number of intraepithelial lymphocytes (IEL) as well as the presence of crypt hyperplasia and villus atrophy. These changes are denoted as Grades I, II and III (IIla: mild atrophy; IIIb: marked atrophy; IIIc: total atrophy) reflecting the increasing severity of mucosal changes. ${ }^{(12)}$ With WLE, the Sn of diagnosing mild or patchy villous atrophy is an abysmal 59\%. ${ }^{(13)}$ Narrow-band imaging (NBI), however, has the ability to differentiate normal and abnormal mucosal patterns in the oesophagus and stomach as well as in the small intestine. The evidence so far has shown NBI to be superior to conventional WLE in the evaluation of duodenal villi in CD and suspected malabsorption. ${ }^{(14,15)}$ The benefits of NBI include facilitating targeted biopsy of abnormal mucosa, thereby reducing costs and complications, which may in turn mitigate the need to perform random biopsies.

There are only a few studies that examined the diagnostic ability of NBI with magnification endoscopy (NBI-ME) to detect villous changes specifically in CD. In one study of 44 patients by De Luca et al, NBI-ME detected villous atrophy in all 17 patients with established CD. In contrast, WLE was only able to identify seven of the patients. Hence, the Sn of NBI-ME was much better than that of WLE (100\% vs. $41 \%) .{ }^{(15)}$ An earlier study from Australia assessing villous atrophy using NBI-ME showed that this novel tool displayed $\mathrm{Sn}$ of $93.3 \%$ and Sp of $97.8 \%$ in patients with suspected CD. ${ }^{(16)}$ These studies only enrolled patients with a high pretest probability of CD who had either malabsorption or positive serological tests.

${ }^{1}$ Gastroenterology Department, Lyell McEwin Hospital (Northern Adelaide Local Health Network), ${ }^{2}$ School of Medicine, University of Adelaide, Australia Correspondence: Dr Doreen Koay Siew Ching, Consultant, Gastroenterology Department, Lyell McEwin Hospital, Haydown Road, Elizabeth Vale, 5112, South Australia, Australia. Doreen.koay.s.c@singhealth.com.sg 

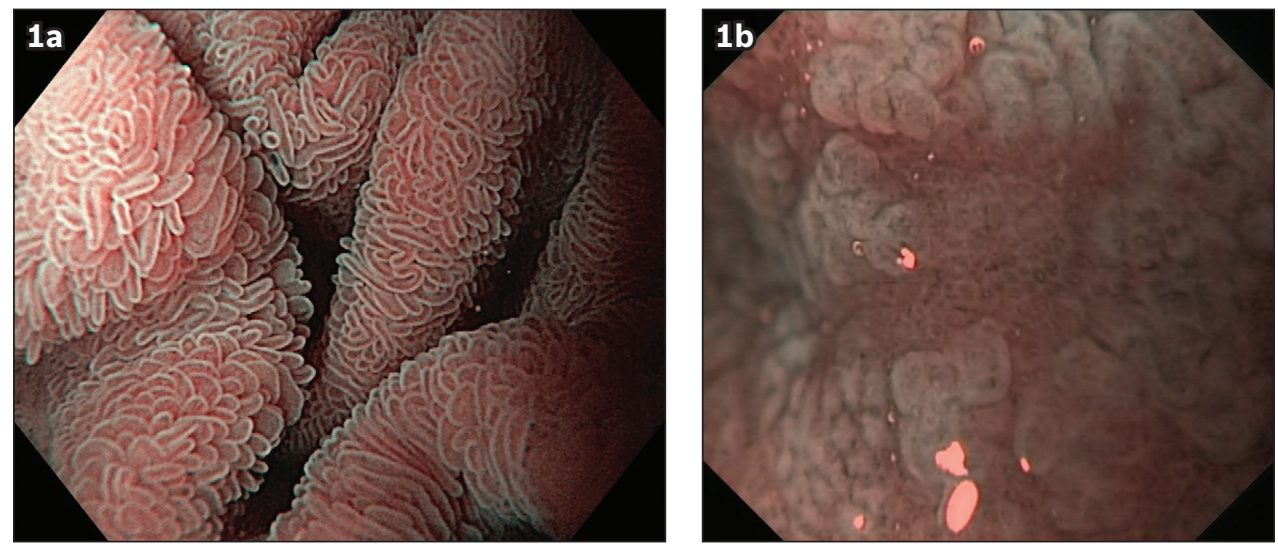

Fig. 1 Water immersion with narrow-band imaging and magnification images show $(a)$ normal villi $(\times 60)$ and $(b)$ partial-to-total villous atrophy $(\times 60)$.
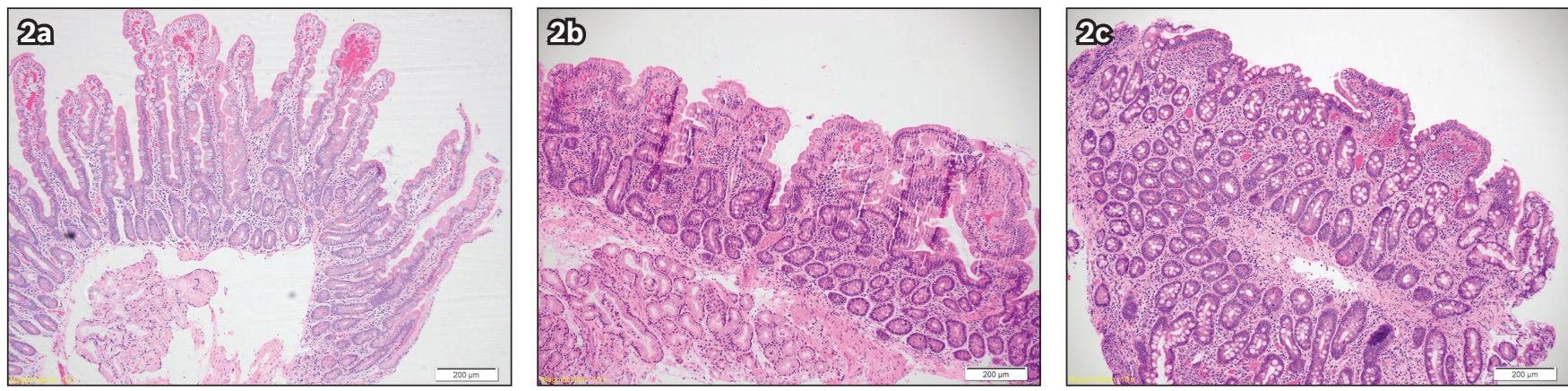

Fig. 2 Histology images show (a) normal villi (Haemotoxylin \& eosin [H\&E], ×10); (b) partial villous atrophy (H\&E, ×10); and (c) total villous atrophy (H\&E, ×10).

NBI-ME can be used with the water immersion technique (i.e. NBI-ME-WIT), enabling even greater enhancement of the mucosal/villi architecture (Fig. 1). Visualisation of villi with the water immersion technique (with WLE) has been shown to have a higher positive predictive value (PPV) and Sp for diagnosis of $\mathrm{CD} .{ }^{(17)}$ To date, there have been no studies examining the diagnostic ability of NBI-ME-WIT for duodenal diseases in general. Hence, the aim of this study was to determine the performance of NBI-ME-WIT in detecting villous atrophy in all patients presenting for upper gastrointestinal (GI) endoscopy irrespective of the baseline risk of $\mathrm{CD}$. We also calculated the hypothetical cost saved if a biopsy-avoiding approach was used.

\section{METHODS}

This was a single-centre prospective study to assess the performance of NBI-ME-WIT in detecting villous atrophy. Written informed consent was obtained from each patient prior to upper GI endoscopy. All patients who underwent upper GI endoscopy between July 2008 and July 2015 were evaluated for eligibility.

Half an hour prior to the procedure, patients were given a drink of a mucolytic agent, $\mathrm{N}$-acetylcysteine, and a defoaming agent, simethicone, mixed with water (total $50 \mathrm{~mL}$ ). The procedures were performed either using a $160 \mathrm{Z}$ (pre-year 2011) or a $190 \mathrm{HQ}$ endoscope (Olympus Australia Pty Ltd, Melbourne, Victoria, Australia) and a high-definition television monitor (OEV181H; Olympus Australia Pty Ltd, Melbourne, Victoria, Australia). Both endoscopes had a magnification capability with a range of 60-115 times. A transparent distal attachment cap was used for all procedures.
The duodenum was further examined with NBI-ME-WIT only after the examination with WLE in the oesophagus, stomach and duodenum did not reveal the cause of anaemia or dyspepsia. The duodenal lumen was filled with approximately $50 \mathrm{~mL}$ of water and views obtained with the endoscopic tip immersed under water. The transparent hood prevents water from escaping and ensures that a good fluid seal is obtained between the tip of the scope and the duodenum. If there was excessive peristaltic activity that interfered with obtaining optimal, crisp views, an antispasmodic agent, hyoscine-N-butylbromide (10-20 mg), was administered. Villous patterns were predicted based on a previous validated classification. Targeted duodenal biopsies were then taken irrespective of what was predicted on NBI-MEWIT. The duodenal villous patterns observed using NBI-ME-WIT were compared with the final histopathological analysis (Fig. 2) and used to calculate the Sn, Sp, PPV and negative predictive value (NPV) of NBI-ME-WIT in detecting villous atrophy. Patients whose histology showed Marsh Grade I (increased IEL) on histology were excluded from the study, as NBI-ME is not technically capable of detecting this.

Data was analysed to calculate the Sn, Sp, PPV and NPV of NBI-ME-WIT in detecting villous atrophy. Hypothetical cost saved by using a biopsy-avoiding approach was also calculated.

\section{RESULTS}

A total of 124 patients were evaluated. The process of patient enrolment is shown in Fig. 3. 83 (67\%) patients were female, with a mean age of 46 (range 18-82) years. The most common indication for investigation was abdominal pain $(n=48,39 \%)$, 


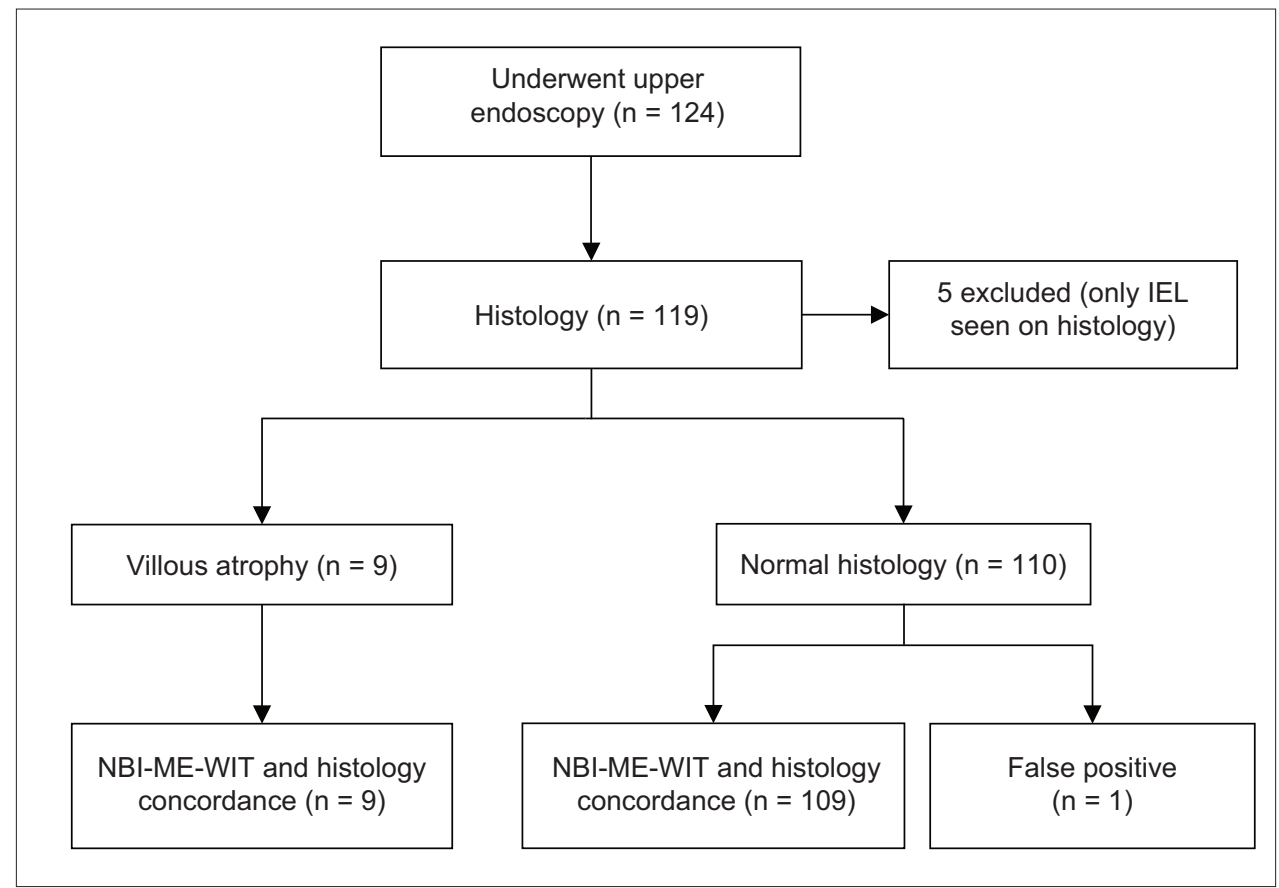

Fig. 3 Flowchart shows the study enrolment and outcome. IEL: intraepithelial lymphocytes; NBI-ME-WIT: narrow-band imaging with magnification endoscopy and water immersion technique

followed by anaemia $(n=44,35 \%)$, chronic diarrhoea $(n=24$, $19 \%$ ) and dyspepsia $(n=8,6 \%$ ) (Table I). An average of 3.5 (range 1-7) biopsy bites were taken for each patient.

Villous atrophy was present in 9 (7\%) patients; eight had patchy villous atrophy, while one had total villous atrophy. NBI-ME-WIT detected all nine patients. The Sn, Sp, PPV and NPV of NBI-ME-WIT in detecting villous atrophy were $100.0 \%$, $99.1 \%, 90.0 \%$ and $100.0 \%$, respectively. The final histological diagnosis in patients who had villous atrophy was duodenitis $(\mathrm{n}=3,33.3 \%)$, coeliac disease $(\mathrm{n}=2,22.2 \%)$, reactive changes $(\mathrm{n}=2,22.2 \%)$ and Crohn's disease $(\mathrm{n}=2,22.2 \%)$. Raised IEL counts were found in five patients. A review of medical records and inquiry from the patients via a phone conversation revealed that none of them had been diagnosed with $C D$. Three were diagnosed with irritable bowel syndrome, one had spontaneous resolution of symptoms, and another patient had spontaneous partial improvement of symptoms with no diagnosis given. Importantly, none of these patients reported any symptoms of malabsorption or long-term sequelae.

Taking into account the cost of a single biopsy forceps (AUD 17) and Medicare Benefits Schedule (Australia) for pathology (AUD 140), a proposed biopsy-avoidance strategy could have saved AUD 18,055 in this cohort of patients.

\section{DISCUSSION}

Although CD prevalence has been rising over the last decades, diagnostic tools remain limited, leading to delays in diagnosis for this group of patients. Endoscopic markers using standard WLE have high $\mathrm{Sn}$ for diagnosing mucosal abnormalities, although it can vary from $50 \%$ to $94 \% .{ }^{(10,18,19)}$ This figure drops further for subclinical CD and patchy villous atrophy. ${ }^{(13,20-22)}$ Image-enhanced endoscopic technologies such as NBI-ME-WIT may play a vital
Table I. Baseline characteristics of the study population $(n=124)$.

\begin{tabular}{|ll|}
\hline Variable & No. (\%)/mean \\
\hline Age $(\mathbf{y r})$ & 46 \\
\hline Gender & \\
\hline Male & $41(33)$ \\
\hline Female & $83(67)$ \\
\hline Indication & \\
\hline Abdominal pain & $48(39)$ \\
\hline Anaemia & $44(35)$ \\
\hline Chronic diarrhoea & $24(19)$ \\
\hline Dyspepsia & $8(6)$ \\
\hline
\end{tabular}

role in identifying abnormal changes and subsequently directing targeted biopsies.

$\mathrm{NBI}$ is a novel endoscopic imaging technique that enables characterisation of subtle mucosal changes. In NBI, the bandwidths of the blue and green filters used are narrowed, forming a narrowed wavelength of light. This light is maximally absorbed by haemoglobin, enhancing the visualisation of capillary microvasculature. It also has a relatively more superficial penetration compared to conventional white light, thus accentuating surface microstructure.

In this study, we showed that NBI-ME-WIT had high Sn (100\%) and Sp (99.1\%) for detecting villous morphology suggestive of $\mathrm{CD}$. In our previous study, we examined the villous morphology using $\mathrm{NBI}$ and high magnification without water immersion on a sequence of videos. The overall Sn and Sp to correctly distinguish the presence or absence of villi in that study were $93.3 \%$ and $97.8 \%$, respectively. The Sn and Sp in differentiating partial from total villous atrophy were $83.3 \%$ and $100 \%$, respectively. ${ }^{(16)}$ The study group was, however, small and comprised only 21 patients. 
In another recent study of 105 patients, of which 51 patients had CD, Goswami et al demonstrated that NBI-ME has Sn, Sp, PPV and NPV of $95.0 \%, 90.2 \%, 91.2 \%$, and $94.2 \%$, respectively, for diagnosing altered villous morphology. ${ }^{(14)}$ The clinical benefits of the NBI-ME-WIT technique include its ability to facilitate targeted biopsy of abnormal mucosa, which may otherwise be missed on WLE. This technique also has the potential to decrease procedure time and reduce cost and complications if the need for a biopsy can be reduced.

The main strength of our study is that it is a novel case-finding study. We examined the capability of NBI-ME-WIT in identifying $\mathrm{CD}$ patients among any presenting patient who required an endoscopy after a cause of Gl bleeding was ruled out (i.e. no ulcers/varices, dieulafoy lesions, erosions, etc). In the literature to date, no such study has been performed.

This study has a few limitations. All procedures were performed by a single expert endoscopist, which affects its generalisability. Another potential limitation includes the exclusion of patients with raised IEL counts. The prevalence of raised IEL count was reported to be as high as $5.4 \%$ in the general population. ${ }^{(23)}$ Although raised IEL counts can be an early manifestation of CD (Marsh Grade I), this finding is not specific for $C D$, which is only one of many possible causes. Other causes of raised IEL counts include Helicobacter pylori infection and the use of medications such as nonsteroidal antiinflammatory drugs, as well as small bowel bacterial overgrowth and systemic autoimmune disorders. Regardless of the cause, a raised IEL count can cause diarrhoea and weight loss. If it is associated with $\mathrm{CD}$, clinical signs of anaemia and dermatological manifestations can often be seen. Natural history, long-term consequences and the role of IEL in CD diagnosis remain uncertain.

In conclusion, NBI-ME-WIT is a specific and sensitive tool to recognise and accurately diagnose villous atrophy. Biopsies can be avoided in patients with normal-sized villi, which may decrease the overall cost of the procedure.

\section{REFERENCES}

1. Green PH, Cellier C. Celiac disease. N Engl J Med 2007; 357:1731-43.

2. Di Sabatino A, Corazza GR. Coeliac disease. Lancet 2009; 373:1480-93.
3. Anderson RP, Henry MJ, Taylor R, et al. A novel serogenetic approach determines the community prevalence of celiac disease and informs improved diagnostic pathways. BMC Med 2013; 11:188.

4. Cranney A, Zarkadas M, Graham ID, et al. The Canadian Celiac Health Survey. Dig Dis Sci 2007; 52:1087-95.

5. Taylor E, Dickson-Swift V, Anderson K. Coeliac disease: the path to diagnosis and the reality of living with the disease. J Hum Nutr Diet 2013; 26:340-8.

6. Rubio-Tapia A, Hill ID, Kelly CP, Calderwood AH, Murray JA; American College of Gastroenterology. ACG clinical guidelines: diagnosis and management of celiac disease. Am J Gastroenterol 2013; 108:656-676; quiz 677.

7. Brocchi E, Corazza GR, Caletti G, et al. Endoscopic demonstration of loss of duodenal folds in the diagnosis of celiac disease. N Engl I Med 1988; 319:741-4.

8. Jabbari M, Wild G, Goresky CA, et al. Scalloped valvulae conniventes: an endoscopic marker of celiac sprue. Gastroenterology 1988; 95:1518-22.

9. Stevens FM, McCarthy CF. The endoscopic demonstration of coeliac disease. Endoscopy 1976; 8:177-80.

10. Smith AD, Graham I, Rose JD. A prospective endoscopic study of scalloped folds and grooves in the mucosa of the duodenum as signs of villous atrophy. Gastrointest Endosc 1998; 47:461-5.

11. McIntyre AS, Ng DP, Smith JA, Amoah J, Long RG. The endoscopic appearance of duodenal folds is predictive of untreated adult celiac disease. Gastrointest Endosc 1992; 38:148-51.

12. Oberhuber G, Granditsch G, Vogelsang H. The histopathology of coeliac disease: time for a standardized report scheme for pathologists. Eur J Gastroenterol Hepatol 1999; 11:1185-94.

13. Oxentenko AS, Grisolano SW, Murray JA, et al. The insensitivity of endoscopic markers in celiac disease. Am J Gastroenterol 2002; 97:933-8.

14. Goswami A, Dadhich S, Bhargava N. Use of narrow band imaging in assessing duodenal villous atrophy. Indian J Gastroenterol 2014; 33:440-4.

15. De Luca L, Ricciardiello L, Rocchi MB, et al. Narrow band imaging with magnification endoscopy for celiac disease: results from a prospective, singlecenter study. Diagn Ther Endosc 2013; 2013:580526.

16. Singh R, Nind G, Tucker G, et al. Narrow-band imaging in the evaluation of villous morphology: a feasibility study assessing a simplified classification and observer agreement. Endoscopy 2010; 42:889-94.

17. Gasbarrini A, Ojetti V, Cuoco L, et al. Lack of endoscopic visualization of intestinal villi with the "immersion technique" in overt atrophic celiac disease. Gastrointest Endosc 2003; 57:348-51.

18. Bardella MT, Minoli G, Radaelli F, et al. Reevaluation of duodenal endoscopic markers in the diagnosis of celiac disease. Gastrointest Endosc 2000; 51:714-6.

19. Mauriño E, Capizzano H, Niveloni S, et al. Value of endoscopic markers in celiac disease. Dig Dis Sci 1993; 38:2028-33.

20. Tursi A, Brandimarte G, Giorgetti GM, Gigliobianco A. Endoscopic features of celiac disease in adults and their correlation with age, histological damage, and clinical form of the disease. Endoscopy 2002; 34:787-92.

21. Achkar E, Carey WD, Petras R, Sivak MV, Revta R. Comparison of suction capsule and endoscopic biopsy of small bowel mucosa. Gastrointest Endosc 1986; 32:278-81.

22. Dickey W, Hughes D. Prevalence of celiac disease and its endoscopic markers among patients having routine upper gastrointestinal endoscopy. Am J Gastroenterol 1999; 94:2182-6

23. Walker MM, Murray JA, Ronkainen J, et al. Detection of celiac disease and lymphocytic enteropathy by parallel serology and histopathology in a population-based study. Gastroenterology 2010; 139:112-9. 